\title{
LVII. Critical velocities for electrons in helium
}

\section{Frank Horton \& Ann Catherine Davies}

To cite this article: Frank Horton \& Ann Catherine Davies (1920) LVII. Critical velocities for electrons in helium, Philosophical Magazine Series 6, 39:233, 592-603, DOI: 10.1080/14786440508636072

To link to this article: http://dx.doi.org/10.1080/14786440508636072

$$
\text { 曲 Published online: } 08 \text { Apr } 2009 .
$$

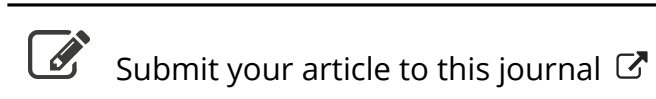

$$
\text { 山 Article views: } 4
$$

Q View related articles $\sqsubset$

4 Citing articles: 2 View citing articles 진 


\section{$\left[\begin{array}{ll}592 & ]\end{array}\right.$}

LVII. Critical Velocities for Electrons in Helium. By Frank Honton, Professor of Physics in the University of London, and Ann Catherine Davies, Royal Holloway College, Englefield Green *

$\mathbf{I}^{\mathrm{N}}$ $\mathrm{N}$ a recent paper $\dagger$ the authors have shown that the first critical electron velocity for the normal helium atom is 20.4 volts, and that an electron with this velocity is able to produce radiation from the atom on collision with it. It was also shown that when the velocity of the impacting electron is increased to 25.6 volts, it is able to produce ionization by collision. This value of the minimum ionization velocity is lower than that predicted for the normal helium atom by Bohr's theory, according to which the electron velocity necessary for the removal of one electron from a helium atom is 2.13 times the electron velocity required to ionize an atom of hydrogen, while the electron velocity necessary to remove both electrons from a helinn atom at a single collision is $6 \cdot 13$ times the hydrogen ionization velocity 'Taking the values $e=4 \cdot 774 \times 10^{-10}$ E.S.U., $e / m=1.767 \times 10^{2}$ E.M.U., and $h=6547 \times 10^{-27} \mathrm{gm}$. cm. ${ }^{2} / \mathrm{sec}$, Bohr's theory gives 13.54 volts as the hydrogen ionization velocity. The two critical ionization velocities for helium, calculated from Bohr's theory, are therefore 28.84 volts and 83.00 volts respectively, the difference between these two velocities, viz. $54 \cdot 16$ volts, being the electron velocity which, according to the same theory, would be required for the removal of the second electron from a helium atom which had already been ionized.

After the conclusion of the experiments described in our earlier paper, we investigated the effects of collisions between helium atoms and electrons having considerably greater velocities, with a view to obtaining some further evidence of the validity of Bohr's assumptions, by determining accurately the velocity at which both electrons are removed from the normal helium atom at a single collision. This investigation presented considerable difficulty on account of the fact that, at the higher critical velocities, increases of radiation or of ionization have to be detected in the presence of the effects which occur at lower velocities. Our experiments showed that to obtain a degree of accuracy at all comparable with that attained in the earlier research, it would be necessary to use an electron stream in which all the electrons have the same velocity, so that the breaks in the current curves,

* Communicated by the Authors.

† Proc. Roy. Soe. A. vol. xev. p. 408 (1919). 
which indicate critical points, may be well marked. We hope to make further experiments using an apparatus in which the electrons in the primary stream are sorted out by means of a magnetic field, so that all those which enter the ionization chamber have the same velocity. The results contained in the present paper, which can only be considered as approximate, are given for comparison with the recently published work of Franck and Knipping*. These authors have obtained values in agreement with those which we found for the minimum electron velocities for the production of radiation and of ionization in helium, and, in addition, have produced evidence that a third type of collision, resulting in an increase of ionization, occurs when the velocity of the impacting electron is raised to 79.5 volts. In determining this latter point the difficulties we have mentioned, of obtaining really marked bends in the current curves with high electron velocities, were also experienced.

The arrangements used in the present experiments have been described in detail in earlier papers, but the method can be understood by reference to the diagrammatic representation in fig. 1. Electrons from the glowing filament $F$

Fig. 1.

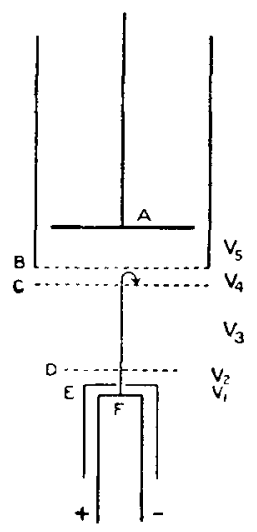

were accelerated towards the surrounding electrode $\mathrm{E}$ by a difference of potential, $\mathrm{V}_{1}$, which was constant during any particular series of observations. Those electrons which passed through the small hole in the centre of the top of $\mathrm{E}$, were further accelerated by the potential difference $V_{2}$, which was varied during the experiments. The potential

* J. Franck and P. Knipping, Phys. Zeits. xx. p. 481 (1919). 
difference $V_{4}$, between the gauzes $B$ and $C$, was in the reverse direction to $V_{1}$ and $V_{2}$, and was sufficiently great to prevent any of the electrons from reaching $B$. The usual path of the electron stream is thus indicated by the arrow in the diagram. A represents the collecting electrode which was connected to a sensitive electrometer for measuring the currents due to ionization or radiation. Its distance from the gauze $B$ could be varied over a range of several centimetres. In most of the experiments the apparatus employed was that which we had used in our previous investigation with helium. but the gauze $C$ had been added, the distance between ( $\mathrm{C}$ and $\mathrm{D}$ ) being about $1 \mathrm{~cm}$. The later experiments were made with a sliwhtly modified form of apparatus in which the distance between $\mathrm{C}$ and $\mathrm{D}$ was abont $3 \mathrm{~cm}$, and this apparatus was afterwards used for experiments with argon; a complete description of it is given in the Proceedings of the Royal Soeiety, A. vol. xcvii. p. 1. The helium used was carefully purified before being stored, and during the observations was slowly circulating through the experimental tube, entering through a U-tube containing carbon and immersed in liquid air. The pressure of the gas. during the experiments was varied according to the particular point which was under investigation.

In considering the points at which breaks might bo expected in the current-potential difference curves for high values of the accelerating potential difference, we started from the facts already established, that radiation is produced from helium by impacts of e ectrons with 20.4 volts velocity, and ionization by impacts of electrons with $25 \cdot 6$ volts velocity. Under suitable conditions of pressure it should be possible to obtain curves giving indications of the production of radiation and of ionization by the second, third, etc. inelastic collisions of electrons with helium atoms. The particular points on the curve at which indications are to be expected will depend upon whether the distance over which the accelerating potential difference is applied is large or small compared with the mean free path of an electron in the gas. For instance, in order that a radiation curve should show increases of radiation at multiples of the resonance velocity ( 204 volts), the pressure must be so high that most of the electrons in the stream from the filament make several collisions in traversing the distance over which the accelerating potential difference is applied, so that for the range of velocities used the potential fall along a free path is not likely to exceed the resonance voltage. Under these conditions, very little, if any, ionization will take 
place at 25.6 volts, because so few of the electrons can acquire the necessary energy*. It would thus seem that the conditions necessary for the increases of radiation to occur to any large extent at multiples of 20.4 volts, and the conditions necessary for the indication of ionization at $25 \cdot 6$ volts, are mutually exclusive.

If the pressure is so adjusted that the distance over which the accelerating voltage is applied is about the same as, or is smaller than, the mean free path of an electron in the gas, the points at which bends, other than those at $20^{\circ} 4$ volts and $25 \cdot 6$ volts, occur in the current-potential difference curves will depend upon the length of path traversed by the electrons before their energy is reduced by the retarding field to a value below that at which they can make inelastic collisions. If this distance is large compared with the mean fret path, so that the electrons make several collisions with gas atoms, the current-potential difference curve may be expected to indicate an increased production of radiation at $(25 \cdot 6+20 \cdot 4=)$ $46^{\circ} 0$ volts, and an increased production of ionization at $(25 \cdot 6+25 \cdot 6=) 51 \cdot 2$ volts. Increases of radiation and ionization should occur again at 71.6 volts and at 76.8 volts, respectively, and so on. If, however, this distance is about equal to the mean free path or is less than this, the currentpotential difference curves would not be expected to show breaks at the vélocities just mentioned, bui would be expected to do so when collisions between electrons and gas atoms oceurred which resulted in the transference, at a single impact, of a larger amount of energy than that corresponding to $25 \cdot 6$ volts, such, for instance, as would result in both electrons being removed simultaneously from the helium atom, at which point the curve should indicate an increase of ionization. Such collisions would not be prevented from occurring, even when the path traversed by the electrons after acquiring the energy corresponding to the applied accelerating potential difference, and before they have had their velocity reduced below $20 \cdot 4$ volts, is several times the mean free path (as in the case when bends at 460,512 , $71 \cdot 6$, and $76 \cdot 8$ volts would be expected); but it is probable that under these conditions, the effect would be to produce a flattening of the curve, instead of an increase of slope, since

* Although, under the conditions mentioned, this statement is probably true of the greater proportion of the electrons in the strenm, certain experiments which are referred to in Proc. Roy. Soc A. vol. xevii. p. 1, seem to show that even when the distance traversed by an electron with $a$ velocity above the critical value is several times the mean free path, some electrons can traverse this distance without suffering an inelastic collision. 
the double ionization of the helium atom at a single collision which, according to Bohr's theory, should oceur at 83 volts, would result in the production of less positive electricity than the expenditure of a smaller amount of energy in removing single electrons from three helium atoms, with the consequent loss of $25 \cdot 6$ volts velocity at each of the three successive collisions.

In most of the experiments described in this paper, the gauzes $\mathrm{D}$ and $\mathrm{C}$ were at the same potential, so that the electrons, after being accelerated by the fields $V_{1}$ and $V_{2}$, traversed the distance between $\mathrm{D}$ and $\mathrm{C}$ without suffering any reduction of velocity except that resulting from their collisions with helium atoms. The electron stream was prevented from spreading laterally in this space by means of a magnetic field, parallel to the axis of the tube, applied by passing a current of abont 2 amperes through a coil of many turns of wire wrapped round the experimental tube. Since there is no difference of potential between the gauzes $\mathrm{D}$ and $\mathrm{C}$, the detection of ionization depends upon the diffusion of the positive ions out of this space. Those which pass through the gauze $C$ will be accelerated by the field $V_{4}$ (which retards the electron stream) and driven towards the gauze B. Some of these will pass through B and reach the collecting electrode $A$, provided that the field between $B$ and A does not oppose them, or is insufficient to turn them back. Experiments showed that this method gave satisfactory results, and that, with the gas-pressure suitably adjusted, ionization could always be detected when the accelerating potential difference was increased beyond the ionization value.

Some of the curves obtained, which illustrate the differences in the effects occurring at different gas-pressures, are given in figs. 2, 3, and 4 In these curves (and in the others given in this paper) the electron velocities, expressed in equivalent volts, are those of the swiftest electrons present in the stream from the filament. The method of obtaining these velocities for given values of the applied accelerating potential difference is explained in our earlier paper ${ }^{*}$. The curve in fig. 2 was taken under such conditions that any positive ions produced would be prevented from reaching the collecting electrode, so that the current due to radiation was examined alone. The gas pressure during this experiment was about $1.5 \mathrm{~mm}$., which should cause the electrons to make several collisions with helium atoms while traversing the distance over which the accelerating potential difference was applied. It will be observed that the curve indicates an 
increased production of radiation at the second and third multiples of the resonance potential difference.

Fig. 2.

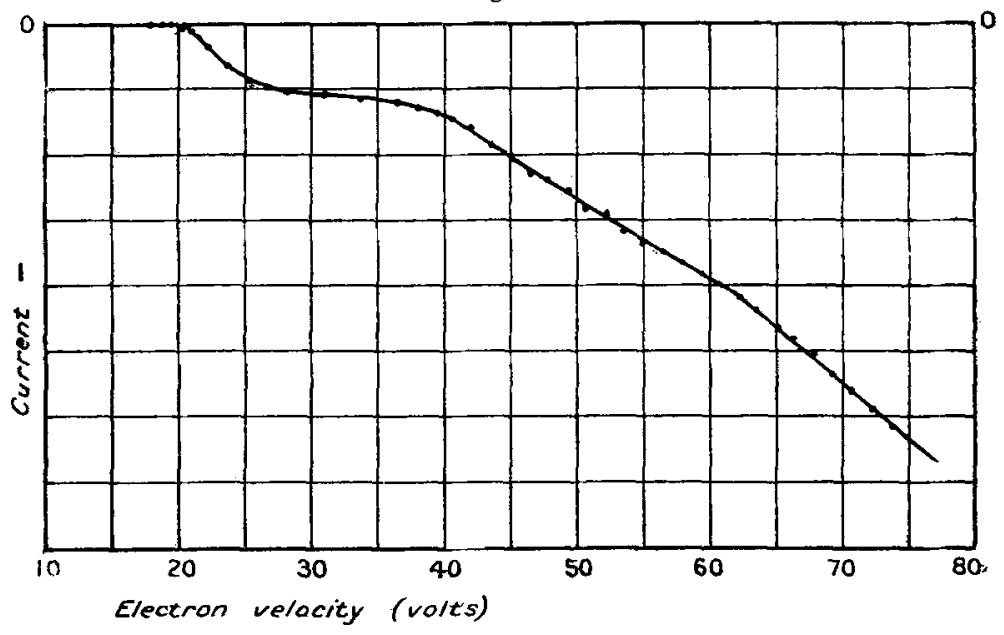

Fig. 3.

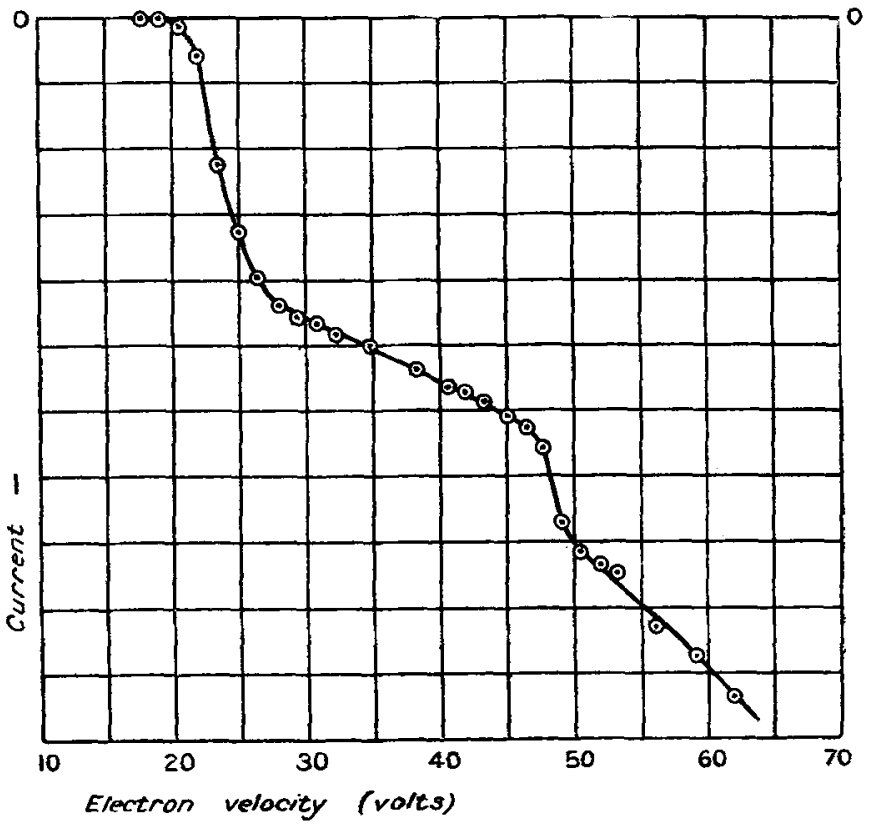

Fig. 3 also represents a series of observations of the variation with the velocity of the electrons, of the current. 
due to radiation alone. These observations were taken at a pressure of $0.49 \mathrm{~mm}$., so that most of the electrons would not make more than one collision while traversing the distance over which the accelerating potential difference was applied. This curve indicates an increased production of radiation, not at the second multiple of the resonance velocity but at $(25 \cdot 6+20 \cdot 4=) 46 \cdot 0$ volts.

In the curve of fig. 4 the pressure is still lower, being only $0.17 \mathrm{~mm}$, and at this pressure it is probable that a Fig. 4
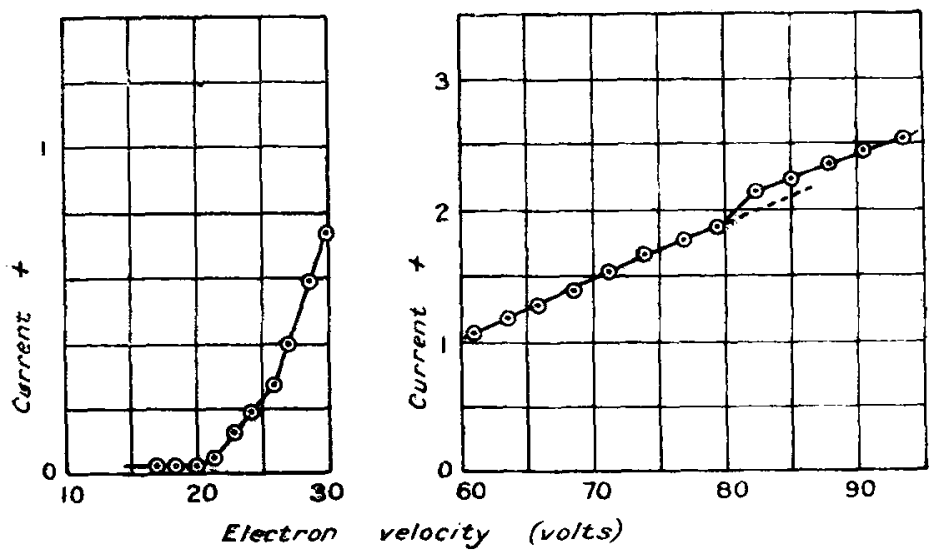

considerable proportion of the electrous pass through the space between the gauzes $D$ and $C$ without making more than one collision with a helium atom, and it is certain that very few, if any, will be able to make three collisions while traversing this distance. For this series of observations the fields were so arranged that radiation and ionization would both cause the electrometer to indicate a positive current. The curve shows a bend at 20.5 volts, another at 25.6 volts, and another one in the neighbourhood of 80 volts. The critical velocity indicated at 80 volts is presumably that at which both electrons are removed from the atom at a single collision. Other series of observations agreed in fixing the critical velocity at which this occurs at about 80 volts, but the difficulty of obtaining the point accurately has already been mentioned. This value should be the sum of the velocities required to remove the two electrons separately from the helium atom. We have shown experimentally that an electron velocity of 25.6 volts is required to remove the first electron, so that the difference between the two experimentally determined values, $i . t$. about 54 volts, is the velocity required to remove the second electron. This 
value agrees with the value deduced from Bohr's theory. In the course of our investigation we obtained direct experimental evidence of the existence of a critical velocity at about this point. It has been mentioned that in most of our experiments there was no difference of potential between the gauzes D and ( , in the space between which most of the ionization occurred. In view of this fact, when the ionization velocity has been exceeded, positive ions will be distributed throughout the path of the primary electrons, and it is possible that at a suitable pressure and with a sufficiently intense electron stream, collisions take place between ionized helium atoms and electrons with considerable velocities. If a sufficient number of such collisions occurred, the current-potential difference curve would be.expected to show a rise when further ionization resulted from these encounters. An example of a curve in

Fig. 5.
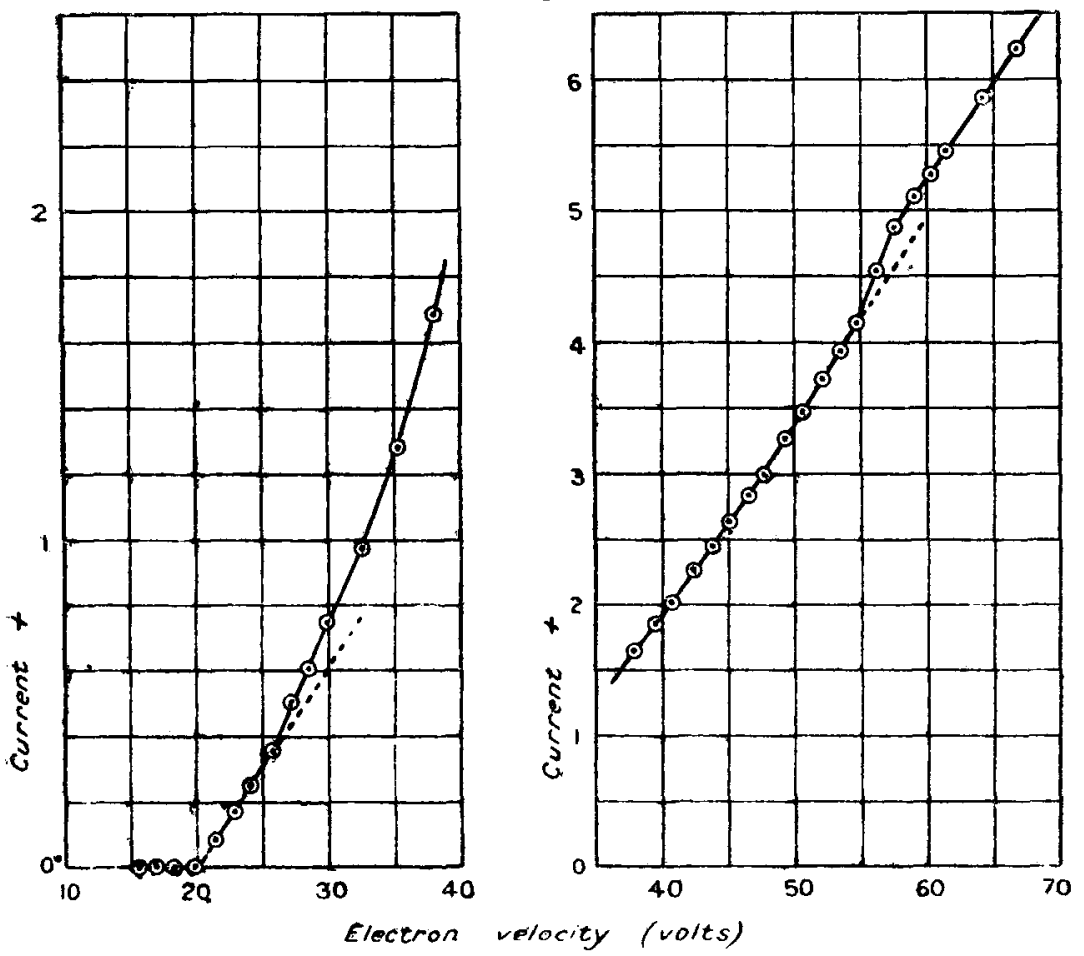

which an increase of current was obtained, which could not be attributed to the production of radiation or ionization by the successive collisions of an electron with different helium atoms, is given in fig. 5 . This curve was obtained 
at a pressure of $0.35 \mathrm{~mm}$., and show's an increase of positive current at about 55 volts. It is therefore possible that this bend does indicate the production of ionization as a result of encounters between ionized helium atoms and electrons. having this velocity, and that it thus affords direct experimental evidence of the correctness of Bohr's assumptions as applied to the helium atom which has lost one electron.

If collisions occur hetween ionized atoms and electrons, it follows that the number of collisions between electrons and normal helium atoms will be reduced by the recombination which probably results when the velocity of the impacting electron is lower than some critical value. On this account, with the intense electron stream which must be used in order that the electron collisions with ionized atoms may be sufficiently numerous for the removal of the second electrons from these ionized atoms to cause a rise in the current curve, it is possible that there will be such a small proportion of the electrons making second or third inelastic collisions with normal helium atoms that the curve will show no bends at the electron velocities at which these would normally occur. Thus it was found that when an intense electron stream was used (as in obtaining the curve of fig. 5), the current curve showed a rise at about 55 volts but no bends at 46.0 volts or at 51.2 volts, whereas in curves taken at a similar pressure when a smaller electron current was employed, the former point was not marked but bends at relocities corresponding to multiple collisions were obtained. The absence of the bends at 46.0 volts and at $51 \cdot 2$ volts under the conditions of experiment of fig. 5 may thus be an indication that a large number of collisions with ionized helinm atoms is occurring, a condition which is essential to the interpretation we have given of the rise at 55 volts. If this interpretation is correct, it might be expected that there would be some indication of an increase in the measured positive current at an electron velocity, lower than 55 volts, which would correspond to the production of radiation from the ionized helimm atom by its collision with an electron. On Bohr's theory such radiation would require for its production an electron velucity of $\frac{3}{4}$ of $54 \cdot 16$ volts $=40 \cdot 62$ volts. The ratiation corresponding to this potential difference would be of such high frequency as to be able to ionize some of the normal helium atoms present, and it is therefore uncertain whether its production would show in the current-potential difference curve as an increase of radiation or as an increase of ionization, in a case where the arrangement of the electric fields made it possible to discriminate between these two 
effects. The electron velocity at which the bend in the curve is to be expected (40.6 volts) is almost identical with the point at which, under suitable pressure conditions, an increase of radiation should be produced by the second collisions of electrons with normal helium atoms, but the two effects can be distinguished, since the conditions which favour the occurrence of ionization, and which therefore favour the production of the $40^{\circ} 62$ volt radiation, are those under which increases of radiation at multiples of the resonance velocity would not be likely to occur to any appreciable extent.

In considering the possibility of detecting the production of a new type of radiation or of fresh ionization from the collisions of electrons with helium atoms which have already lost one electron, the effects of recombination must be taken into account. It is conceivable, for instance, that recombination takes place at all encounters between positively charged helium atoms and electrons with velocities below that necessary for the production of radiation from the charged atom-40.62 volts on Bohr's theory. This being the case, an increased positive current should be measured by the electrometer at $40 \cdot 62$ volts on account of the reduction at this point in the amount of recombination occurring. Whether in this case an increased positive current would also be obtained when the electron velocity is raised to that necessary to remove the second electron from the ionized atom is uncertain, and would depend upon whether the ionization then produced were more than that which the $40 \cdot 62$ volt radiation was producing from the normal helium atoms.

Again, it is possible that an electron which gives rise to 40.62 volt radiation itself recombines with the positively charged helium atom with which it collides. In this case an increased positive current should be obtained when the critical velocity for ionization of the positively charged helium atom is reached, since the encounters would then result in the production of doubly charged helium atoms instead of neutral ones. As the precise conditions under which recombination of a colliding electron and a positively charged helium atom on which it impinges occurs are unknown, it is impossible to predict how the existence of critical velocities at $40 \cdot 62$ volts and $54 \cdot 16$ volts for this atom should be indicated. The curve in fig. 5 shows a bend which we have attributed to the further ionization of the positively charged helium atom, but no bend at all in the neighbourhood of 40 volts. By altering the experimental arrangements we Phil. Mag. S. 6. Vol. 39. Nc, 233. May 1920. 2 R 
were able to make a bend show up at this latter point under conditions when it could not be due to a second radiating collision at twice the resonance velocity. A series of observations in which this result was obtained are plotted in the curve of fig. 6 . In taking these observations a potential

Fig. 6.

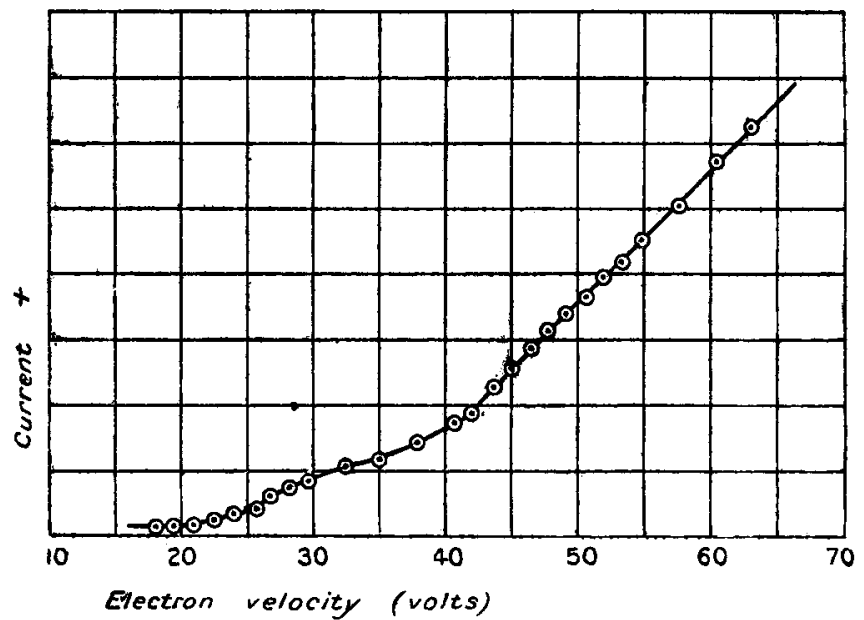

difference of 7 volts was maintained between the gauzes $D$ and $\mathrm{C}$ (which in most of the earlier experiments had been at the same potential) in such a direction as to drive positive ions towards the collecting electrode $\mathrm{A}$, and the potential difference $V_{5}$ was in the direction for radiation, as well as ionization, to give a positive charge to the electrometer. The gas-pressure $(0.170 \mathrm{~mm}$.) was too low for it to be likely that the bend at 41 volts is due to multiple collisions, and it may therefore be taken to indicate a new critical velocity at this point. An upward bend at about 41 volts was obtained in several curves taken under conditions similar to those stated. In some experiments at still lower pressures (about $001 \mathrm{~mm}$.) taken with the final field $V_{5}$ reversed, a bend in the opposite direction was obtained, showing that radiation was produced at this point, and that at this low pressure its photoelectric effect on the cylinder was greater than the ionization it produced in the gas.

The curves in figs. 5 and 6 may thus be interpreted as supporting the view that at 41 volts and at 55 volts electron collisions with positively charged helium atoms occur, which result in an increased positive current being measured by 
Method of solving rroblems in Conduction of Heat. 603

the electrometer, as would be expected if radiation and further ionization, respectively, were produced from the ionized atoms at these velocities. Although these results are in agreement with the predictions of Bohr's theory, it is clear that the method of experimenting affords no absolute proof that the observed increase of current does result from such collisions.

In connexion with the increase of current observed at 80 volts, and interpreted as being due to the removal of both electrons from a helium atom at a single electron collision, it might be suggested here also that for some lower electron velocity a single collision should result in the removal of one electron and the displacement of the other to an orbit of greater radius, thus giving both ionization and radiation. Some experiments by Ran * seem to show that collisions producing an effect of this kind do occur under certain conditions. On the basis of Bohr's theory, the electron velocity at which such an effect would be expected is 25.6 volts $+\frac{3}{4}$ of $54 \cdot 16$ volts $=66 \cdot 22$ volts. We were unable to obtain any evidence of this effect in our experiments.

The results described in this paper agree with those of Franck and Knipping in showing that the double ionization of the helium atom results from collisions between helium atoms and electrons having about 80 volts velocity, but differ from theirs in indicating bends in the current curves at 41 volts and 55 volts which appear to be due to the production of radiation and of further ionization, respectively, from the helium atoms which have already lost one electron.

I,VIII. Bromwich's Method of solving Problems in the Conduction of Heat. By Prof. H. S. Carslaw, Sc.D.†

1. T $\mathrm{N}$ his paper-" Examples of Operational Methods in Mathernatioal Physics," Philosophical Magazine, No. 220, April 1919-Bromwich advocates the use of socalled operational methods, following Heaviside, in electrical and other physical problems, and illustrates his method by the solution of various questions, including some in the Conduction of Heat. In an earlier paper (Proc. London Math. Soc. (Ser. 2) xv. p. 401, 1917) he discussed Heaviside's work, and confirmed it by the aid of contour integrals.

The object of the present paper is to illustrate by some

* H. Rau, Würzburg Plys. Med. ges. Ber. Feb. $19 \mathrm{4}$.

+ Communicated by the Author.

$2 \mathrm{R} 2$ 